\title{
La irenología en la formacion de la dignidad humana
}

\section{Irenology in the formation of human dignity}

\author{
Karla Sáenz López ${ }^{1}$ \\ María Leonor Ramos Morales ${ }^{2}$
}

Resumen: El concepto de la Irenología es relativamente nuevo, surge después de la segunda guerra mundial, es importante retomarlo porque nos da un enfoque de cómo educar desde la familia, la escuela y la sociedad con la finalidad de restaurar o regenerar el tejido social. Se mencionan las habilidades principios y elementos como estrategia hacia una cultura de paz, porque desarrollar las habilidades de las personas para poder convivir de forma pacífica con otras personas y consigo mismos son la base para que el ser humano pueda preservar su dignidad, que es el pilar de los derechos humanos

Palabras clave: Irenología, cultura de paz, dignidad humana.

Abstract: The concept of Irenology is relatively new, it arises after the Second World War, it is important to resume it because it gives us an approach of how to educate from the family, school and society with the purpose of restoring or regenerating the social fabric. The principles and elements are mentioned as a strategy towards a culture of peace, because developing the skills of people to be able to coexist peacefully with other people and with themselves is the basis for the human being to preserve their dignity, which is the pillar of human

rights

Keywords: Irenology, culture of peace, human dignity

\footnotetext{
${ }^{1}$ Doctora en Ciencia Política por la Universidad Complutense de Madrid; Nivel I del Sistema Nacional de Investigadores del Consejo Nacional de Ciencia y Tecnología ; Profesora de la Facultad Contaduría Pública y Administración de la Universidad Autónoma de Nuevo León https://orcid.org/0000-0002-7279-0342.

${ }^{2}$ Doctora en Métodos Alternos de Solución de Conflictos por la Universidad Autónoma de Nuevo León; Maestra con Especialidad en Métodos Alternos de Solución de Conflictos por la Facultad de Derecho y Criminología de la Universidad Autónoma de Nuevo León: Licenciada en Educación por la Universidad Pedagógica Nacional; Docente de Facultad de Derecho y Criminología De la UANL. Candidata a Investigadora Nacional por el Sistema Nacional de Investigación. ramos.leonor@gmail.com https://orcid.org/0000-0002-2875-9399
} 


\section{Introducción}

En este capítulo se desarrolla desde la concepción de la Irenología, su origen y como se fue desarrollando a través de la historia. Es un concepto relativamente nuevo por que se empieza emplear después de la segunda guerra mundial, desde los griegos y los romanos ellos ya utilizaban el vocablo eirene y pax respectivamente. Es importante mencionar que se realiza un camino para mostrar como los principales agentes de cambio como son la familia, la escuela y la sociedad pueden influir para poder regenerar la paz social. Se plantean tres escenarios que desde la escuela pueden influir para la formación de individuos capaces de socializar en forma armónica y evitar la violencia, se sugieren desarrollara habilidades, principios y elementos que favorecen en individuo la convivencia en un estado de armonía dentro de una sociedad cambiante.

Se intenta relacionar la familia, la escuela y la sociedad con la intención de hacer visible la necesidad de restaurar la paz social, a través de la influencia de estos agentes de cambio. Las personas que tengan claro que la socialización y la construcción del sentido de la identidad los va a llevar a ser individuos con más confianza para poder establecer una comunicación positiva, tener una formación en cultura de paz que les favorezca a poder desarrollarse dentro de diferentes contextos que están en una sociedad. Individuos que reaccionan ante la adversidad sin importar la clase social. Individuos solidarios para mejorar la convivencia y regenerar el tejido social.

Se trata de ver la responsabilidad, la ética y la moral construirse en diferente escenario de formación y socialización y que da la posibilidad de respetar las diversidades y se fundamenta en la convivencia como expresión de autonomía, libertad y dignidad humana. La dignidad es de difícil conceptualización, porque una sola definición no describe a plenitud la idea compleja creada a partir de la palabra; pero podemos definirla como una cualidad del ser humano que tiene con el solo hecho de existir. No se adquiere ni se asigna. Está relacionada con la libertad, el libre albedrío, la autodeterminación y el origen divino de la existencia.

Entender la dignidad, permite comprender las normativas que regulan las relaciones humanas, empezando por la Declaración Universal de los Derechos Humanos de 1948, que declara en el preámbulo y en su artículo primero, a la dignidad como la base filosófica que justifica dicha declaración, el Pacto Internacional de Derechos Civiles y Políticos de 1966 es otro ejemplo de normativa que basa su justificación en la dignidad humana. También es parte importante de las discusiones en el derecho penal.

Si partimos de que la dignidad es inalienable a la existencia humana, entonces porque debemos protegerla y preservarla, Pogge, $2011^{3}$ hace mención de que en la misma Declaración Universal es contradictorio primer decir que es una condición de la familia humana y párrafos después declara que para garantizar la dignidad hay que cumplir requisitos

${ }^{3}$ Pogge ,Thomas. 2011. Dignidad y justicia social. Dianoia. Pags. 3.12
Revista Juris Poiesis - Rio de Janeiro. Vol.22-n ${ }^{\circ} 28,2019$, pg.208- 228. ISSN 2448-0517 Rio de Janeiro, 29 de abril de 2019. 
previos. Entonces alguien que no tiene esos requisitos previos, puede perder la dignidad que inicialmente no es posible perder.

(Pogge, 2011) nos menciona tres dimensiones en las que la dignidad se puede perder, la primera es cuando un ser humano está en un estatus social por debajo de otro, como es el caso de humillaciones, golpes, gritos, violencia, ya sea en matrimonios, trabajos, desplazados, encarcelados, pacientes, ancianos, alumnos menos populares. La segunda es cuando la persona se pone a si misma por debajo de las demás, cuando no cuida su propio cuerpo en higiene, alimentación, ejercicio, y finalmente se percibe como disminuida frente a otros, teniendo un sufrimiento auto infligido, y la tercera es relacionada al estado mental de la persona en donde puede estar sujeta a bajas pasiones, sentimientos negativos, pereza, holgazanería o problemas mentales que hacen que la persona tenga un sufrimiento por no poder realizar acciones complejas o tener fuerza de voluntad.

Si pensamos en cuantas personas viven alguna de las tres dimensiones antes mencionadas, entenderíamos que la dignidad humana está en un grave problema a nivel global.

Es significativo conocer el origen del concepto que tiene un origen religioso y cómo evolucionó al ámbito civil y moral, así como entender cómo se forjó su posición en el centro del deber ser en las relaciones entre personas, grupos y naciones.

\section{Concepto de Irenología}

La irenología es una ciencia comparativamente reciente, se empieza a mencionar después de la segunda guerra mundial, donde alcanza un auge en el continente europeo. Uno de los primeros investigadores que la impulso fue Johan Galtung ${ }^{4}$, uno de los máximos y más conocidos referentes de esta corriente, sociólogo y matemático noruego, autor de una significativa serie de artículos y libros referentes a la sociología de la paz, y precursor en 1959 del primer instituto para la investigación, el International Peace Research Institute of Olso. Una de sus grandes virtudes, es la asociación de los fenómenos de la violencia y el conflicto. Johan Galtung afirma que la paz puede ser aprendida y desarrolla el tema desde ahí, como un proceso ético y positivo de aprender a abordar los conflictos de un modo distinto al violento.

El término irenología tiene un origen etimológico del griego eirene "paz" y logos "ciencia, estudio o tratado", entendiéndose por irenología como la ciencia que estudia o trata la paz. El término irenología es definido por J.G. Starke (citado en Cabello, 2012) ${ }^{5}$ lo define

"como el cuerpo de teorías, conceptos, hipótesis, principios, generalizaciones, leyes generales, deducciones y proposiciones formuladas sobre el tema de la paz, dirigidos a identificar fuerzas y condiciones que, desde una perspectiva positiva, ayudan al

\footnotetext{
${ }^{4}$ Galtung, Johan. (1998). Cultura de paz y gestión de conflictos, Primera edición, Paris 1998, 403 páginas.

${ }^{5}$ Cabello Tijerina, Paris, Alejandro. (2012). La mediación como política social aplicada al fortalecimiento de la cultura de paz en México y España. Tesis doctoral en intervención social y mediación. Universidad de Murcia.
} 
mantenimiento de un régimen pacífico $y$, desde la óptica negativa, pueden ser quebrantadoras de este".

La presente propuesta se encuadra en la perspectiva positiva sobre la paz, asumida con fines a posibilitar varios escenarios para tocarse el tema de la irenología con el objetivo de promover en la sociedad capacidades ordenadas y dialógicas frente a diferentes situaciones que sobrelleven a conductas y actos pacíficos, salvados con asertividad. Esto también en relación con los planteamientos de Cabello (2012), al manifestar que uno de los principales logros de la irenología, fue concebir al fenómeno paz, como la circunstancia óptima de la sociedad y por su empeño en la búsqueda de soluciones pacíficas a los conflictos.

Toh comenta que los conflictos que se originan en cualquier lugar o sociedad suelen poseer vínculos externos o interdependencias que demandan asimismo de una ocupación o de enfoque crítico. En efecto, casualmente, los impulsos ejercitados desde el externo pueden ser tan eficaces que "no tomar en cuenta" podría comprimir arduamente las posibilidades de resolución del conflicto local y de la construcción de la paz. (p.1026). Planea que los conflictos suelen tener enlaces que incluso pueden nacer convenientemente desde el lenguaje, donde la única forma de adjudicarse y prevalecer es a través de un enfoque rigurosamente crítico.

Mockus $^{7}$ afirma Convivir es llegar a vivir juntos entre distintos sin los riesgos de la violencia y con la expectativa de aprovechar fértilmente nuestras diferencias. El reto de la convivencia es básicamente el reto de la tolerancia a la diversidad y esta encuentra su manifestación más clara en la ausencia de la violencia... (p.20). A través de la presente propuesta promover la sana convivencia en la sociedad y es por ello, desde la irenología se quiere beneficiar el corriente social, contribuyendo secundariamente a mejorar la convivencia en la sociedad.

López (2006) ${ }^{8}$ La irenología posee una representación multidisciplinar, creando informes de los distintos estudios sobre la paz, a saber: estudios prácticos, críticos y constructivistas. De estas orientaciones el que nos interesa es encuadrar en la publicación crítica de la paz, en la representación de analizar y discutir diversas situaciones que giran en torno a la paz. Según la teoría de conflictos de Galtung (1998), se muestra la necesidad de provocar en el progreso de todo ser humano la resolución pacífica de conflictos como principio ético de cualquier sociedad que se muestre a superar la guerra, de igual manera Galtung identifica dentro del diagnóstico de cualquier conflicto que la violencia estructural también aporta una gran importancia.

Mientras tanto Savater (1998) está de acuerdo con lo que plantea de Galtung empleando un sentido extenso e incrementado, invitando a que se debe emplear la razón como inseparable y en esencia de los conocimientos que se llevan a cabo, cuando se habla de paz en el caso a

\footnotetext{
${ }^{6}$ Toh Hin, Swee. (2002). -La educación para aprender a vivir juntos. Construcción de la paz y educación para la paz: Experiencias locales, reflexiones globales-. Perspectivas, vol. XXXII, N. ${ }^{\circ}$ 1, marzo 2002.

${ }^{7}$ Mockus, Antanas. (1994). -La educación para aprender a vivir juntos, convivencia como armonización de ley, moral y cultura-. Perspectivas, vol. XXXII, N. ${ }^{\circ}$ 1, marzo 2002. P. Ver: Boulding Kenneth. Paz estable entre las naciones: un proceso de aprendizaje. Revista Cuadernos de Ciencias Económicas y Empresariales, N. ${ }^{\circ}$ 26, 199

${ }^{8}$ López Gómez, Óscar «Pas e Sosyego» Un argumento de acción política en la castilla bajomedieval. Medievalismo: Boletín de la Sociedad Española de Estudios Medievales, ISSN 1131-8155, N. 16, 2006, págs. 41-72
} 
tratar dentro del contexto social,...educación es formar seres humanos, y los seres humanos somos ante todo racionales. La razón no es una disposición meramente automática, sino un logro social, posibilitado por unas capacidades naturales, evolutivas, etc. (...) razonar es una disposición natural basada, o para nosotros fundada, en el uso de la palabra, en el uso del lenguaje; y el uso del lenguaje es lo que nos obliga a interiorizar nuestro papel social. (p.1).

De acuerdo con las referencias anteriores se pretende acercar a la sociedad a la comprensión del concepto de irenología, mediante el abordaje de la resolución de conflictos y a la sana convivencia, fortaleciendo la capacidad para analizar situaciones y exponer argumentos en torno a la paz.

\section{El origen de la irenología}

La paz ha estado presente a lo largo de la historia y encontramos diversos indicios en diferentes culturas y épocas. Se tiene diferentes formas de definir la paz, de acuerdo con la lengua, al campo semántico y expresiones que complementan y que se trasmiten de generación en generación Muñoz y López (2004) ${ }^{9}$. La capacidad de tener conductas pacíficas y permiten la evolución de las diferentes sociedades en distintas épocas.

Los seres humanos son conflictivos y pacíficos de forma natural y en situaciones de violencia pueden reaccionar de acuerdo con lo aprendido. Po lo que la paz y la violencia siempre ha existido, la sociedad, la civilización, el aprendizaje, compartir, los colectivos han sido factores que dan el origen a los seres humanos.

En Grecia el vocablo que se utilizaba para describir a la paz era eirene y se consideraba como sinónimo de homonoia que significa armonía. Con estas palabras los griegos simbolizaban la paz social en la que vivían dentro y fuera de su territorio. Se usaba el término eirene para describir la paz social y como la armonía mental y espiritual. Para los griegos la exploración de la perfección converge en sentimientos serenos y tranquilos.

Los romanos utilizaban el termino pax para reseñar a la paz y su significado es semejante a usado por los griegos eirene. ${ }^{10}$ La pax es la expresión latina que tiene su raíz en pak, que significa establecer una reunión para resolver por medio de un acuerdo el conflicto de las partes. Los romanos cuando organizaban convenciones o acuerdo del daba el nombre de pax, porque este concepto está profundamente relacionado con la imagen de mantener y respetar "lo legal"- es conocida como la paz de la ley y el orden ${ }^{11}$. La pax romana fue un axioma de paz en referencia de" ausencia de violencia según la ley" y no como justicia, bienestar, correspondencia e igualdad para todos. En el mundo occidental se concibe la paz en un sentido negativo, es decir la separación de violencia. Entonces el significado más común de paz es la ausencia de violencia o guerra.

\footnotetext{
${ }^{9}$ Muñoz Muñoz, F., López Martínez, M. (2004) Historia de la paz, en B Molina Rueda, \& F. Muñoz, Manual de Paz y Conflictos (págs. 42-65) Granada: Eirene

${ }^{10}$ Cabello Tijerina, P., Vázquez Gutiérrez, R. (2018) Cultura y educación para la paz. Una perspectiva transversal. México Tirant lo Blanch. Pág. 45

${ }^{11}$ Ledrach, J.P. (2000) El abecé de la Paz y los Conflictos. Educación para la paz. Madrid Catarata 
El origen de la paz está también relacionada con las religiones occidentales como el judaísmo, el cristianismo y el islam y las orientales el hinduismo, el taoísmo y el budismo. ${ }^{12}$ En nuestra sociedad el cristianismo y el catolicismo hablan de los proverbios de la no-violencia y el amor como fundamento que le da origen a estas religiones. La religión cristiana se basa en los valores de amor, armonía, protección, correspondencia y de equidad que es una propuesta a la resolver conflictos no utilizando la violencia. En las religiones orientales la paz se cree como la calma y la moderación íntima de las personas y su ambiente. En este contexto las religiones orientales y occidentales se enmarcan en una paz positiva.

\section{México y la Irenología}

En México se conoce muy poco la Irenología, por lo que es muy importante su difusión por medio de las academias formadoras de la cultura y educación para la paz. México es un país que ha vivido la violencia principalmente por el narcotráfico, las instituciones gubernamentales se han enfocado a la seguridad creando cuerpos de reacción fuertemente armados que ayudan a combatir y disminuir la violencia, pero recordemos que combatir la violencia con violencia, esto hace que se genere más violencia.

Hay que promover los valores como la paz, crear nuevas oportunidades de empleo con salarios dignos que le permitan a los ciudadanos tener condiciones de vida básicas, un servicio médico de calidad, viviendas que les permita al os ciudadanos tener una sana convivencia en el interior como al exterior, política pública de calidad donde se fortalezca el respeto, la responsabilidad social y se fortalezca la cultura de paz para que los ciudadanos puedan vivir juntos con dignidad y en un contexto social agradable.

\section{Concepto de paz}

No existe un concepto ecuménico y exacto de lo que significa la paz, por lo que exploraremos algunos conceptos. Proverbialmente la paz se refiere a la ausencia de guerra y para que la paz pueda permanecer tendría que existir primero un enfrentamiento belicoso. Tendríamos que eliminar este concepto vacío, inconsistente e impreciso y crear uno nuevo para evadir la violencia y desarrollara capacidades, habilidades y destrezas para la transformación de los conflictos en oportunidades de aprendizaje, de cambios, de comunicación e intercambio de nuevas formas de resolver conflictos.

Paulo Fiare ${ }^{13}$ cree que la paz es una realización de las potencialidades humanas, que la paz se crea y se edifica con la construcción continua de la justicia social. La paz no se puede separar de la justicia, está ligada en el contexto nacional, internacional, social e interpersonal, es decir, la paz es la independencia, la equidad y la conformidad.

Se pude concluir que la paz es la evolución de los conflictos de una forma creativa, que los conflictos nuca van a dejar de existir lo interesante es como los vamos a resolver, para ello

\footnotetext{
${ }^{12}$ González Ballesteros, L., Sainz Martin. A., Hens Muñoz, M., \& Sánchez Corral. (1993) Signos y cultura de violencia. Una investigación en el aula. Córdoba: Servicios de publicaciones de la Universidad de Córdoba

${ }^{13}$ SODePaz (27de marzo de 2019). Cultura de paz. Obtenido de Cultura de Paz: http:/www.culturadepaz.info/home.php Revista Juris Poiesis - Rio de Janeiro. Vol.22-n²8, 2019, pg.208- 228. ISSN 2448-0517 
debemos tener comprensión, imaginación, comunicación, colaboración y empatía, para para fomentar en la sociedad la cultura de paz.

\section{Regenerar la paz social}

La formación en la corriente social debe ser uno de los objetivos del proceso de enseñanza y aprendizaje de las ciencias sociales, facilitando a la sociedad capacidades de colaboración activa para poder actuar en su entorno y en la sociedad en general; de igual manera los individuos deben desarrollar el pensamiento social para poder estar al tanto de inmiscuirse su contexto y con sus acciones poder hacer parte de la metamorfosis social.

Visualizar se realiza una simple transmisión de conocimientos sería desconocer al individuo como agente pensante, como si su mente fuera un equipaje en donde se guarda o impone las ciencias, facilitando el proceso de enseñanza y aprendizaje como solo una admisión de ideas, saberes o información que se da o se transmite.

Campanario y Otero $(2000)^{14}$, plantean que es necesario tener en cuenta: ...que los individuos mantienen un vinculado diverso de ideas anteriores o premeditación sobre los contenidos científicos que casi siempre son erradas y se reconoce voluntariamente que estas ideas anteriores son uno de los elementos clave que, deben tenerse en cuenta como condición necesaria (aunque no suficiente) para un aprendizaje significativo. (p.156).

La sociedad actual debe asumir la encomienda de formar a niños, niñas y jóvenes para que puedan resolver los conflictos que se les van presentando en su vida cotidiana, así como tomar medidas para resistir las influencias que reciben de su medio social a fin de que no renuncien a la primera dificultad o fracaso. La sociedad actual súplica que los individuos no se limiten solo a adquieran conocimientos, sino que formen poblaciones competentes para vivir y convivir en sociedad, jueces de su entorno, capacitados para apropiarse de su ambiente y de orientar sus intereses, se está exigiendo una enseñanza más fundada y diatriba, una enseñanza realmente pedagógica que supere la rutina pequeña, para establecer concretamente un cambio.

\section{Hacia una educación de calidad para restaurar la dignidad humana}

Cada individuo desde que nace empieza a socializar y con ello la adquisición de conocimientos, de la personalidad y descubrimiento del medio o contexto social donde se desarrolla. De la niñez a la edad adulta va pasando por períodos de sus vidas y la coexistencia con diferentes grupos sociales, con esto ira afianzando su carácter y personalidad y encontrara así, una formación personal y profesional. En estas etapas de la vida conocerá normas, comportamientos y actitudes que le servirán para conocer los diferentes ámbitos de

\footnotetext{
${ }^{14}$ Campanario, Juan Miguel y Otero, José C. (2000). Más allá de las ideas previas como dificultades de aprendizaje: las pautas de pensamiento, las concepciones epistemológicas y las estrategias metacognitivas de los alumnos de ciencias. Grupo de Investigación en Aprendizaje de las Ciencias. Departamento de Física. Universidad de Alcalá de Henares. Alcalá de Henares. Madrid.
}

Revista Juris Poiesis - Rio de Janeiro. Vol.22-n²8, 2019, pg.208- 228. ISSN 2448-0517 
socialización y donde desarrollara los valores y hábitos adquiridos a través del crecimiento como persona.

Las principales instituciones que influyen en la instrucción de la persona son la familia, la escuela y la sociedad, es donde se adquieren las normas, los valores, las reglas para poder desenvolverse en la sociedad y se van heredando de generación en generación y es una constante para que los individuos determinen diferentes influencias que van a determinar el destino de sus vidas. Es importante reconocer la paradoja de la dignidad, porque si bien, es inherente a la existencia humana, las personas no nacemos con la información necesaria para manejarnos a nosotros mismos y nuestras relaciones, de tal forma, que tanto los individuos como los grupos sociales requieren ser capacitados en el desarrollo de habilidades para desarrollar la paz en sus relaciones y en el entorno.

\subsection{La Familia primer agente de enseñanza}

La primera instauración en la vida de un individuo es la familia y esta adquiere la responsabilidad de formar personas con valores, reglas y comportamientos que se van desarrollando a lo largo de la vida de cada individuo. Es el núcleo familiar donde cada miembro va a adquirir las habilidades para resolver los diferentes conflictos que se van presentado y será la familia que le brinde el apoyo necesario para que adquiera las herramientas para que pueda vivir una vida plena y de sana convivencia.

En la familia cuando se establecen normas de cooperación, respeto, asignación de tareas y responsabilidades, los conflictos, económicos, familiares, sociales serán parte de toda la familia y como tal deben de responder cada uno de acuerdo con el grado de responsabilidad que se va adquiriendo. ${ }^{15}$

La familia es el origen en donde el niño asimila la noción de ser humano, de ser persona, en donde se enseña la educación, donde asimilan los hábitos fundamentales que van a cumplir toda su vida, como por ejemplo el control de emociones, la limpieza, alimentarse etc. también aprenden elementos básicos acerca de lo modelos de afectividad del ser humano, el concepto de madre y padre; sexo, mujer y hombre y todo eso en un momento decisivo de su crecimiento y desarrollo, los primeros años de su vida.

En la casa donde aprendemos firmemente, pues la educación es una emprendedora inapreciable; se podría decir eterna, por ello debemos verlo como el área de variadas influencias educativas que interactúan entre sí. Los elementos formativos en la familia son: el padre, la madre, los abuelos, los hermanos, los primos, amigos del hogar, el servicio doméstico, los medios de comunicación e información, etc. En los hogares los padres no son siempre dos, a veces es una sola persona, de común la mujer, quién se ve forzada a realizar los dos papeles: padre y madre. Los padres representan dos papeles elementales; el padre la autoridad mientras que la madre la sensibilidad. En un tercer nivel el niño, es el que representa el maestro, el del saber.

\footnotetext{
${ }^{15}$ García Longoria Serrano, M. (2009). Educación Familiar. El aprendizaje de la resolución de conflictos. En J. Paradas Navas, \& J. González Ortiz, La familia como espacio educativo (págs. 161-173) Murcia: Espigas

Revista Juris Poiesis - Rio de Janeiro. Vol.22-n²8, 2019, pg.208- 228. ISSN 2448-0517 
A los padres les corresponderían enseñar el hacer, el quehacer, autoridad, saber y afectividad que son los cinco conceptos básicos de la educación inicial del niño: Ese hacer y quehacer están mancomunados a la formación, la disciplina, el orden, la creación, el invención, la mesura; los padres tienen el compromiso de ayudar a este nuevo ser a afianzase el mundo físico y social, a que asimile a descubrir e identificar los objetos, los seres y a sí mismo, conduciéndolo a una formación y desarrollo de la razón; enseñarles que somos humanos , a asumir críticamente y con creatividad, a enseñarles que la vida tiene un sentido auténtico: nacimiento, matrimonio, divorcio, muerte; que nuestros actos son voluntarios pero comprometidos, enseñarles a tomar decisiones frente a los distintos problemas y conflictos y que existen alternativas; que hay decisiones que se deben tomar ene 1 momento y que otras pueden postergar.

Lo importante y esencial es que lo que se enseña a los hijos en los primeros años es intensamente significativo porque si bien la conducta es transformable, esos primeros años se deja una huella imborrable. ${ }^{16}$.El hogar es una fuente constante de aprendizaje y se enseña a los hijos aun cuando no tengamos la intención de hacerlo; por que actúa de modo eficaz el factor de imitación y a continuación de modelaje.

Un niño aprende de sus padres los dos modelos de seres humanos, el modelo hombre y el modelo mujer. El inconveniente enorme de los padres que son solo mujer, por ejemplo, deriva del hecho de que sus hijos están expuestos a un solo modelo, el propio, mientras el otro se halla ausente. Esta es la consecuencia más importante en los niños de la separación de la pareja. Estamos conscientes de la importancia de la familia y del hogar, pero al mismo tiempo la sociedad presiona ciertos valores que puedan ser formados en la población más vulnerable (niños y adolescentes), la noción del beneficio, la separación por encima de la cooperación y responsabilidad social; el interés por otras culturas extranjeras desfavoreciendo la nacional.

Los niños y los jóvenes necesitan ser educados a partir de la presencia de unos valores claros, bien conformados, con una relación que les de credibilidad. En esto no puede existir el doble discurso, ni la doble vida porque se ceden las vivencias y se viven las creencias. ${ }^{17}$ Es necesario, entonces diseñar un programa en familia ¿Qué?: educando en valores, ¿Dónde?: en todas las áreas y actos de nuestras vidas, principalmente en la familia, ¿Cómo?: por la vía de la reflexión y de la acción. La familia es la que debería liderar la educación y la escuela la instrucción. ${ }^{18}$ Para ello es necesario estimular y empezar a desarrollar las herramientas la responsabilidad histórica que reclama la Patria, la Nación, la Sociedad y dentro de la familia educando en valores para formar las generaciones del futuro.

Los padres y las madres enseñan a los hijos desde que son pequeños a defender sus derechos para que no sean violentados, pero lo más importante que debe enseñar la familia desde el aspecto social es el control de emociones para una mejor integración y sanan convivencia dentro de los grupos sociales en los que se va a desenvolver. Cada hogar tiene sus propios

\footnotetext{
${ }^{16}$ Albornoz, O. (1984). La Familia y la Educación del Venezolano. Ediciones Biblioteca Caracas. Pag 49, 89

${ }^{17}$ Ramos, M. (2000). Programa Para Educar en Valores. La educación que transformará al País. Editorial Paulinas. pág. 55

${ }^{18}$ Opcit 14.
}

Revista Juris Poiesis - Rio de Janeiro. Vol.22-n²8, 2019, pg.208- 228. ISSN 2448-0517 
valores y reglas haca el interior y esto hará fuerte a cada uno de los integrantes para que se puedan adaptar o cambiar dependiendo del contexto social en el que este.

La capacidad de sentir empatía por alguien, de ser justos a la hora de participar, las personas pueden responder de una manera justa, por esto es primordial el papel de la familia para que cada miembro logre interiorizar las formas que existen para negociar, mediar o conciliar área poder manejar necesidades e intereses y solo atacar para ganar.

Los niños pasan de una familia donde adquirieron valores, actitudes, formas de socializar a otra institución en donde se van a adquirir conocimientos y se van a integrar a grupos en el área escolar donde hay rangos, roles y reglas disciplinarias de tendrá que adaptarse y cumplir y de la formación que obtuvo en la familia va a depender de su adaptación en el entorno escolar. Por lo que es importante que familia y escuela trabajen en forma conjunta para la formación de la personalidad de cada uno de los individuos y la adquisición de los valores y habilidades sociales para un futuro. Es importante resaltar que el niño no solo es una esponjita que aprende lo que la familia y la escuela le enseñan, es un ser que da respuesta a lo que los padres y maestros le solicitan, sino que también es capaza de dar respuesta también a sus intereses.

De acuerdo con de Velasco Suárez, Ordoñez Acuña Y Roteer Hernández ${ }^{19}$, para que la raíz de la paz este en la familia y su formación de cada uno de sus integrantes recomiendan:

- Promover la comunicación familiar con cada uno de sus integrantes.

- Promover el trabajo colaborativo en el hogar y la ayuda a quien la necesite.

- Participar en mejorar las condiciones físicas del hogar

- Promover la resolución pacífica de los conflictos a través del dialogo en la dinámica familiar.

\subsection{La escuela segundo agente de enseñanza}

Organizar la escuela como atmósfera de formación y socialización implica dos tipos de preocupaciones: la primera se refiere a proporcionar de los manuales pedagógicos, metodológicos y estructurales favorables para la recomendación de los procesos de enseñanza y aprendizaje; desde esta figura la escuela deberá diseñar el objetivo educativo de la formación, el cual por su naturaleza, según Perkins ${ }^{20}$ plantea tres metas fundamentalmente: la retención, la comprensión y el uso activo del conocimiento; la palabra que engloba a estas tres metas, según el mismo autor, es el conocimiento productor, su propósito es el de ser un conocimiento no acumulativo sino actuado, a través del cual se enriquezca la vida de las personas y se les ayude a comprender el mundo y a desenvolverse en él. Por lo que se proponen tres escenarios en donde se puede adquirir el conocimiento.

\footnotetext{
${ }^{19}$ Velasco Suárez, M., Ordoñez Acuña, B., Dávila, M.H., Roteer Hernández. L. (2012) Una educación para la paz. Programa para niños preescolares. México Trillas.

${ }^{20}$ PERKINS, D. (2001). La escuela inteligente. Del adiestramiento de la memoria a la educación de la mente. España: Gedisa. Pág. 18
} 


\subsubsection{Primer escenario: formar y socializar}

Así, a la escuela le corresponderá situar sus prácticas educativas a la preocupación crítica y a la intervención activa de los implicados en la adquisición y comprensión del conocimiento, el cual, es la capacidad que tienen las personas en formación para exponer, enseñar, utilizar y explicar lo aprendido. Se trata de abrir la mente de los estudiantes, de enseñarles a vivir, de cultivarse no sólo de los libros sino de la vida, de causar cambios mentales encaminados a que cada persona aprenda por sí mismo acerca de sí mismo (Colom y Melich, 1997) ${ }^{21}$

Es un propósito de formación que pone sus bríos en hacer que lo asimilado sea significativo y transformador del conducirse el ser humano, que perfecciona una escuela que en sí misma propone una perspicacia del mundo, de sus relaciones y de las organizaciones más correctas para vivir sensiblemente; es así, que se plantea una pregunta por el tipo de institución que se debe estructurar para lograr que dichas opciones sean un hecho.

Es una escuela imaginada como el espacio donde se edifican ambientes ordenados y completos para la evolución del sujeto, mediante los cuales se atenúa el desarrollo del talento como la instrumento más cuidada del hombre para elaborar un conjunto de contemplaciones sobre el mundo real y sobre sus propios ideales idealidades, y al mismo tiempo, dar la razón en las habilidades y destrezas en diversas formas de razonamiento que ilustran y crean situaciones más serenas para el desarrollo humano de los sujetos comprometidos en un proceso de formación. De esta forma la escuela es un espacio de interacción, edificación y mejora de potencialidades necesarias para el entendimiento del mundo, sus relaciones y sus posibles evoluciones.

En la escuela se llevan a cabo intercambios que tienen la intención de adquirir nuevos conocimientos y desarrollara competencias cognitivas, socio afectivas y comunicativas, para llevar al individuo a la edificación de su personalidad y que genere en el sentido de pertenencia. Es aquí, donde se edifica el primer escenario que se conoce como subcomunidad. En este espacio los educandos ayudan a aprender a otros y de acuerdo con sus prácticas, para esto no se necesita la presencia de un profesor, es decir que los alumnos aprenden unos de otros, a través de la interacción, de la formación y de la cultura en la que están inmersos, se van estableciendo relaciones y acceden a diversos contextos.

La interacción es una habilidad humana para entender el pensamiento a través del lenguaje, los gestos y otros medios por el que se manifiestan. Las palabras no hacen posible que se dé una interacción, sino la capacidad de entender los contextos en lo que las palabras, los gestos y los actos que suceden. La interacción involucra a la intersubjetividad, que es la habilidad para entender las mentes de otras personas a través de su lenguaje verbal y no verbal. La intersubjetividad aprueba la negociación de los significados cuando las palabras se pierden, por lo que es en la escuela donde se debe reforzar y construir alumnos componentes para realizar negociaciones y comprender de las acciones diarias planteada en diversos escenarios pedagógicos , y la construcción de la cultura de paz a través del conocimiento y los principios

\footnotetext{
${ }^{21}$ Colom, A.J. Y Melich, J.C. (1997). Después de la modernidad. Nuevas filosofías de la educación._Barcelona: Paidós. Revista Juris Poiesis - Rio de Janeiro. Vol.22-n²8, 2019, pg.208- 228. ISSN 2448-0517
} 
morales y las normas para que no afecten la sana convivencia de los alumnos, sino lo contrario que exista un lugar seguro pata toda la comunidad educativa.

La escuela será así el espacio de cambio, de la comparación continua entre lo que se enseña y lo que se asimila, de la edificación ligada de las experiencias culturales de la afirmación de las igualdades; será el escenario en donde el compromiso educativo se transformarme en un acto, en una experiencia, en un modo individual de entender las necesidades de la comunidad educativa; será el ambiente nativo de la interacción y de la naturaleza de sentidos culturales, sociales y pedagógicos que llamen a los sujetos a vivir bajo el concepto de la dignidad humana, el respeto a las diferencias y la justicia social.

\subsubsection{Segundo escenario: escuela e identidad}

La escuela debe de reconocer al reto de ser formadora y socializadora para la construcción de una sociedad demócrata, múltiple, incluyente, imparcial. Una escuela donde nazca la ética, dónde se deje atrás solo los logros y los resultados educativos, donde los alumnos logren una reflexión como seres humanos, su pasado, el estar aquí y en el ahora y las circunstancias que lo llevaron a vivir el momento. Una escuela donde se vean las relaciones humanas con equidad, inclusión y reconocer que todos son diferentes, crear las condiciones para una sociedad que viva la democracia. Educar es un proceso de cobijo a los que recién llegan, es una experiencia ética que se interesa en formar individuos con sentido de pertenencia a través de las relaciones en la comunidad educativa y que exista reciprocidad donde se logre una relación activa, democrática y negociada que se base en la responsabilidad.

La escuela tiene la misión debería ser educar en la acción y no en la fabricación, es decir, convertir al alumno competente para las funciones que fue formado, que el acto educativo sea un proceso proactivo, predicativo y terminado, es decir que tenga un comienzo y un fin. Este proceso se abre la iniciativa, las novedades y lo impredecible, para que la educación sea una acción novedosa combinada al discurso, al diálogo, y la pregunta el quien eres tú contenga subjetividad, una biografía y una historia. La educación para la acción conforma diversidad, imprevisibilidad, descubrimiento y narración. La narración es la construcción de la identidad o el sentido de pertenencia de un sujeto, de sus historias y cómo actuar en el presente. La construcción de la identidad hace sujetos únicos, hacen valer sus diferencias y esto les permite coexistir en un espacio cotidiano, auténtico y versátil.

Al construir la identidad de los sujetos se elaboran significados de existencia, en su historia se ha conformado una forma individual de sentir, vivir y pensar, también se configuran la forma justificada para convivir y constituir para reconstruirlo como un espacio de calidad de vida, este espacio es vital en las relaciones y la interacción para vivir en sana convivencia.

La vida da diferentes sentidos y dan implícito las actitudes, valores, normas de convivencia y se delimita así, un espacio particular y colectivo y hace que los individuos interactúen por medio del lenguaje, para evidenciar el espacio que ayude a loa humanidad a reconocer de sí mismos y un estado de legitimad en la coexistencia, es decir en paz. 
La escuela con segundo escenario de socialización y desarrollo tiene un terreno protagónico en la difusión y formación de valores en relación con la cultura de la paz. La cultura escolar es un vínculo de valores morales, conocimientos, prácticas, conductas personales, relaciones sociales y aspectos tan determinados como la institución formativa. La escuela deberá ser solo un escenario de adquisidor de conocimientos, de saber y aprendizaje sino también un agente de socializador y tener una intención formativa educar para la vida, es decir, la construcción del individuo para relacionarse con el mundo real.

\subsection{Formación ciudadana}

En el tercer escenario encontramos a la sociedad, juega un papel muy importante en el desarrollo de las habilidades sociales y de convivencia, en donde se viven e intercambian estilos y formas de vida. La sociedad es un factor muy importante en del desarrollo general y complejo de cada persona. Es donde se conocen aspectos positivos y negativos, se reafirman los valores y comportamientos aprendidos en la familia y la escuela.

Cada individuo tiene un sentido de pertenencia a una familia, a una institución educativa y a un grupo social, se van generando subgrupos de personas donde se intercambian intereses y entorno se convive en un entorno fácil y divertido. Sin embargo, toda sociedad es multicultural e inclusive cada individuo es multicultural de acuerdo con sus historias y a pesar de esto se tiene la capacidad de aceptar compartir y convivir. En los subgrupos pueden surgir conflictos o una reacción inadecuada, es por esto por lo que estos tres escenarios la familia, la escuela y la sociedad pueden influir positivamente para poder resolver los conflictos y salir victoriosos de una situación de conflicto.

Se pueden mencionar dos ejemplos normales dentro de una sociedad. Cuando a pesar de pertenecer subgrupos distintos en edad, intereses, necesidades, se logra una solidaridad sorprendente cuando sucede un evento catastrófico como una inundación, un desastre natural, o cuando el todo el grupo de la sociedad tienen un evento que involucre una celebración como el campeonato de un evento deportivo, estas señales que indican apoyo entre las personas estimulan dicha y se activan rápidamente la cooperación responsable y recíproca. Beck $(2003)^{22}$.

Pero no siempre se tienen este sentido de solidaridad en los grupos sociales multiculturales, los elementos de la cultura pueden afectar las relaciones sociales porque cada una de las personas que integran los subgrupos defenderá cuales comportamientos son o no apropiados para ellos. Munduate Jaca \&Medina Díaz (2006) ${ }^{23}$. Cada persona desenvuelve diversas formas de conducta y de reacción dependiendo de la situación que surja en la sociedad o a los grupos sociales a los que pertenecen, es aquí, donde resalta el sentido de pertenencia de cada uno de los individuos, la responsabilidad, la formación, creencias van de acuerdo con la convivencia social y no a la violencia como una forma de resolver los conflictos.

Existen estudios e investigaciones donde se enuncia que la violencia genera violencia, si uno de los tres escenarios socializadores del individuo la familia, la escuela o la sociedad cultivan

\footnotetext{
${ }^{22}$ Beck, A. (2003) Prisioneros del odio. Las bases de la ira, honestidad y violencia. Barcelona: Paidós

${ }^{23}$ Munduate Jaca, L., \& Medina Díaz, F (2006) Gestión del conflicto negociación y mediación. Psicología, Pirámide Revista Juris Poiesis - Rio de Janeiro. Vol.22-n²8, 2019, pg.208- 228. ISSN 2448-0517 
constantemente sentimientos o comportamientos de violencia, las prácticas sociales se afectaran directamente incluyendo formas de comportamiento violentas, como resultado del carácter, personalidad y de su contexto social, estos individuos pueden crear regalas antisociales esto no sería lo único porque en estos enteros se crean jerarquías, expulsiones y crean un poder muy fuerte que afecta al desarrollo de una sociedad armónica .

Existen diversas organizaciones que incitan a las sociedades a tener una sana convivencia en los tres escenarios familia, escuela y sociedad y que todos los elementos con los que cuenten ya sean familiares, culturales y sociales estén relacionados entre sí, porque si un grupo social ejerza una influencia negativa, pueda la familia, la escuela o la sociedad intervenir de forma inmediata y aportar de forma inmediata la experiencia para influir en forma positiva la restitución al grupo social pero de forma positiva y formar a si, ciudadanos responsables, autónomos y con valores, creencias religiosas, políticas, económicas y sociales positivas.

El individuo está en contante interacción y discute constantemente loas reglas formativas, normativas y los principios morales que regulan la convivencia, prueban espacios democráticos que permiten enfrentarse a diferentes explicaciones, actitudes y sentimientos tomados por cada uno de los integrantes del grupo social y justifican su forma de pensar y actuara moralmente ante la sociedad o el contexto en que habitan.

La educación moral se debe abordar dese la perspectiva de dos dimensiones, una dimensión pensada en que se desarrollen estructuras morales que permitan emitir juicios y de argumentos morales y una segunda dimensión donde se desarrollen elementos de orden, de formas de administrar, de pedagogía y didáctico que están en un ambiente de la escuela y que podrá cada individuo desarrollar un currículo para el desenvolvimiento en la sociedad, estas dos dimensiones proporcionan un orden institucional, real y especifico y da respuesta a la formación de los niños, niñas, jóvenes, maestros y adultos, el desarrollo de estas dos dimensiones no es solo un derecho como aparente mente se piensa, es una enseñanza que llevara al desarrollo de una sociedad competitiva y que se pueda vincular entre sociedades distintas.

Formar ciudadanos plenos no solo es un decreto, es tener la tarea de realizar un proceso profundo del desarrollo moral y que la finalidad es que adquiera un pensamiento autónomo y responsables, que es lo que se necesita en los ciudadanos, que ejerza sus derechos pero que también responda a sus obligaciones, que sea participativo y que tome decisiones favorables para la mayoría de la sociedad a la que pertenece.

Es decir, el nuevo modelo de la escuela está basado en que se conozca sus derechos y obligaciones, pero también que la participación en la política, en la ética y la moral que sea un deber ser, que se conecte con organizaciones, instituciones relacionadas con la protección de los derechos y obligaciones y que todos lo tomen con responsabilidad y creen un espacio democrático para su convivencia, aprendizaje y socialización. Con esto no solo se les enseñaría a ser un ciudadano, sino, a ser un ciudadano que viva en inclusión, en equidad y que pueda participar en la creación de normas colectivas de valores y principios de sana convivencia. 
En este sentido, el proceso de la formación de los ciudadanos es para que estén empoderados, con capacidad de reflexionar y replicar, individuos que sea y que otros individuos puedan ser legítimos, reflexivos, críticos y preparados hacer frente a los retos que plantea las sociedades cambiantes. Con pensamiento autónomo, con capacidad de pensar por sí mismos, asumir el resultado de sus actos, reconocer sus derechos y de los demás, posesionarse de sus obligaciones que se integran a la sociedad, esto fu paleteado por Kant $(1993)^{24}$.

De acuerdo con este planteamiento la UNICEF sugiere que la formación ciudadana reconozca varios elementos:

a) La diversidad de las expresiones juveniles e infantiles, que invita a ver individuos con principios y comprensión del mundo diferente. Reconocer la multiplicidad de expresiones encontrar en cada persona la capacidad de construir con otros un mundo mejor. Reconocer en ellos la legitimidad y la expresión, capaces de argumentar las desigualdades, dar una perspectiva estética en relación al mundo.

b) Desarrollar la participación autónoma, una capacidad para autodeterminarse y proponer formas legitimas de interacción y regulación social y normativa que aporten para el enriquecimiento cultural y que las formas de organización sean vigentes.

c) Conocer sus derechos como habitantes de un contexto y sus obligaciones como habitantes responsables y participativos en el contexto social.

La UNESCO es un internacional especializado en el fomento y la promoción de la paz y la seguridad internacional mediante el fortalecimiento de la educación, la ciencia y la cultura ${ }^{25}$.

El objetivo principal de la Unesco es lograr que los Estados miembros incluyan valores como los derechos humanos. La paz, la participación ciudadana y democrática, la tolerancia, la no violencia e el entendimiento intercultural, impulsando la educación para la paz (Vázquez Gutiérrez, 2013) ${ }^{26}$.

\section{Estrategias para desarrollar la cultura de paz y la socialización para mejorar la convivencia y regenerar la dignidad humana}

El diálogo es la herramienta principal para fomentar la cultura de paz, porque es un intercambio de prácticas, opiniones y diferentes formas de ver la vida y lo que se desarrolla. De acuerdo con lo que se observa día a día nos damos cuenta de que las personas no dialogan o conversan correctamente, la conversación se convierte en un dialogo en sordo, en discursos interminables o en altercados sin sentido. Para impedir que el problema avance y se salga de control se establecen habilidades básicas para mejorar la comunicación y evitar que el conflicto tenga un escalda desproporcionada y pueda adquirir dimensiones en las que la paz

\footnotetext{
${ }^{24}$ Kant, I. (1993). La metafísica de las costumbres._Barcelona: Altaya.

${ }^{25}$ Cabello Tijerina, P.A., Carmona Valdés, S.E., Gorjón Gómez, F.J., Iglesias Ortuño, Emilia., Sáenz López, K.A.C., Vázquez Gutiérrez; R.L. (2017) Cultura de paz. México. Editorial Patria

${ }^{26}$ Vázquez Gutiérrez; R.L. (2013). La mediación escolar y las habilidades sociales en los estudiantes de educación secundaria. Un estudio en institutos de la región de Murcia. Comunitania Internacional Journal of Work and Social Sceences, 113.136.
} 
de los individuos se pueda romper y tener consecuencias mayores en las relaciones en la familia, la escuela y la sociedad.

\subsection{Habilidades para la comunicación positiva}

Cabello Tijerina \& otros (2017) Sugieren seis habilidades primordiales para que la comunicación sea positiva:

\section{- Escucha activa}

Una de las principales técnicas para generar un dialogo es la escucha activa, la cual requiere que focalice la atención en el tema, escuche, observe el lenguaje no verbal y comprenda lo que la persona expresa.

De acuerdo con Brian Muldoon (1998) ${ }^{27}$ el proceso de convertir un conflicto en una disputa es fácil, basta con que cada parte cuente la historia del conflicto; pero a la vez, al narrar los pensamientos, reacciones y opiniones se domina el desconcierto; es decir que al escuchar los hechos exteriorizados hay que ser hábil entender los diversos puntos, posiciones y ayuda que se manifiestan identificar el punto esencial que los separa.

Por medio de la escucha activa se genera un clima de confianza y empatía que facilita que se perciba que existe igualdad de situaciones de comunicación y admite comprimir las tensiones existentes y aumentar la cooperación entre las personas involucradas en el dialogo.

Es fundamental retroalimentar demostrando así que se escucha con cuidado, de manera neutral y objetiva; la escucha activa permite analizar lo que se escucha y pensar en lo siguiente que se dirá o hará. Por medio de la de la escucha activa, los sujetos sienten que se les ha escuchado y que se le han entendido y se obtienen las ideas principales de cada individuo.

\section{- Respetar limites}

Respetar limites es tener la capacidad para someter la conversación o de convertirla en un monologo, o decirlo todo, a través de expresiones no verbales. Cuando hay respeto en lo límites de la conversación, se abre la posibilidad de para expresar perspectivas diferentes del conflicto y encontrar soluciones favorables a cada uno de los involucrados. En escaso que uno de los involucrados realizara un comentario negativo lo más conveniente es no actuar a la defensiva, de lo contario se originaria un nuevo conflicto.

${ }^{27}$ Muldoon, Brian. (1998) El corazón del conflicto. Ed. Paidós, España. pág. 63.

Revista Juris Poiesis - Rio de Janeiro. Vol.22-n²8, 2019, pg.208- 228. ISSN 2448-0517

Rio de Janeiro, 29 de abril de 2019. 


\section{- Empatía}

Miquel Regué (2015) ${ }^{28}$ define a la empatía como una reacción vinculada a la situación emocional en la que se encuentra otra persona, lo que siente o lo que puede experimentar. Y en este sentido se pueden diferenciar dos tipos de empatía:

La empatía emocional que es cuando una persona se involucra emocionalmente al ver la situación o los momentos que está pasando otra persona. La empatía cognitiva es cuando la persona es capaz de entender cómo se encuentra una tercera persona. Ser capaz de valorar, la historia, los miedos los sentimientos de cada una de la persona. La confianza de las partes se establece a través de la empatía.

\section{- Respetar opiniones}

Para encontrar a soluciones a los problemas hay que ayudar a las personas que vean otras opciones factibles para la solución del conflicto. Cuando ya se tienen la información que relataron las partes. Es decir, se tiene la información desde el punto de vista de las partes, la tarea es entender es información realizar un reencuadre para que el otro vea o como percibe la situación, es realizar una transformación de significados para que las partes puedan entenderse uno a otro.

Percibir cualquier experiencia o situación desde diferentes aspectos y centrarse en la que resulte más útil, porque una situación pudiera parecer perjudicial y se puede convertir en algo positivo. Se trata de ver cada experiencia desde un punto de vista rentable para convertirla en algo que beneficie a cada una de las partes. Facilita la razón y asimilación de los mensajes que una parte está emitiendo a la otra Sotelo (2103). ${ }^{29}$

\section{- Objetividad.}

La comunicación debe de aclarar la situación que generó el conflicto o desacuerdos, evitándolo pasar al terreno personal.

\section{- Despersonalizar el conflicto}

Al criticar o citar las situaciones propias se centra en la persona y se personaliza el conflicto. La persona se visualiza como contrincante o como el problema, por esto es que el objetivo a este último es "eliminar a la persona".

La fundamentación de la cultura de la paz debe estar fundamentada en el respeto peno de los derechos humanos por lo que continuación se mencionan los principios de la enseñanza y formación en la cultura de la paz.

\footnotetext{
28 Regué Barrera, M. (2015) La química de la empatía. Revista Moleqla No. 12 ISSN 2173-0903 Editado el 21 de marzo de 2015.Universidad Pablo de Olavide, Sevilla, España, p.62.

${ }^{29}$ Sotelo, Elena; Carretero Morales, Emiliano y Ruiz López, Cristina. Mediación y resolución de conflictos: técnicas y ámbitos. Ed. Tecnos. Madrid. Pag. 250

Revista Juris Poiesis - Rio de Janeiro. Vol.22-n²8, 2019, pg.208- 228. ISSN 2448-0517
} 


\subsection{Principios de la enseñanza y formación en la irenología}

Zurbano Díaz de Cerio, (1998). Propone algunas consideraciones importantes.

- Cultivar valores: Enseñar a los alumnos el valor de la justicia. la cooperación, la solidaridad, el desarrollo de la autoestima personal y tomar decisiones.

- Aprender a vivir con los demás: Es aprender a vivir en sociedad con las normas que dicte cada una de las sociedades y esto va a favorecer en desarrollo continuo de la personalidad y en la no violencia.

- Facilitar experiencias y vivencias: Favorecer la democracia en los grupos para la resolución positiva de los conflictos que se puedan presentar en los diferentes contextos que se desarrollen la persona.

- Desarrollo del pensamiento crítico: Manifestar su opinión y no tener miedo de criticar lo que considere criticable.

- Combatir la violencia de los medios de comunicación: Crear una opinión en explícitas diversiones que se manifiestan en el medio del espectáculo.

- Educar en la tolerancia y la diversidad: Reconocer la diversidad y ser tolerante ante los diferentes juicios y opiniones

- Educar en el diálogo y la argumentación racional: Fomentar en las personas que tienen la capacidad de resolver sus controversias por medio del dialogo, la reflexión y el pensamiento.

Se debe trabajar juntos para favorecer que no se presente peligro de una guerra. Asumir el compromiso de educar en favor de la cultura de la paz.

\subsection{Elementos para un cambio social que promueve la dignidad humana}

- Desarrollo de la autoestima: cada individuo será responsable de sus actos y toma de decisiones

- Respeto a las ideas ajenas: ser tolerante a la diversidad de opiniones y reflexionar que pueden llegar un acuerdo por medio del dialogo.

- Reconocer los derechos humanos: la paz es un derecho.

- Aumentar la participación ciudadana: contribuir por el bien común en la sociedad

- Capacidad de análisis crítico: analizar los procedimientos para poder resolver los conflictos que se despende de los sistemas sociales, políticos y económicos.

- Justicia estructural: los seres humanos están inmersos en los conflictos, desarrollara habilidades de resolverlo en forma ética.

- Auto reconocimiento de la dignidad: es reconocer el derecho inalienable de ser tratado con respeto y consideración.

- Determinación de salvaguardar su dignidad: es la firme convicción del individuo de no permitir acciones, palabras o conductas que produzcan reducción a su dignidad.

- Uso congruente del NO: aprender a responder no cuando sea necesario poner límites a las acciones o conductas de otros que dañen al individuo.

- Uso controlado de su capacidad de acción: autodominio para ejercer el poder sin sobrepasar el límite de la dignidad del otro. 
- Renuncia a los beneficios: estar dispuesto a renunciar a cualquier beneficio que provenga de aceptar una pérdida de la dignidad.

- Toma de decisiones: que el individuo tome decisiones consientes basándose en su libre albedrío, libertad y autodeterminación.

La educación es la columna vertebral de la familia y la sociedad, educar a ser responsables, tener una actitud positiva ante la sociedad, desarrollara el diálogo, la aceptación, la diversidad y la cooperación para realizar un verdadero cambio social y poder así regenerar el tejido social y vivir en valores y en paz.

\section{Conclusiones}

La vinculación de la familia, la escuela y la sociedad como agentes importantes para una regeneración social no puede dar como resultado un estado de derecho para todos los integrantes de cada uno de los contextos sociales. Pero, es primordial trabajar en colaboración para obtener resultados positivos. Las grandes sociedades funcionan mejor, si todos sus habitantes promueven ellos valores, los derechos humanos y la participación ciudadana. Si bien es cierto que debemos ser tolerantes y tener la libertad de expresar las ideas y sentimientos, también es cierto que si vinculamos las ideas podemos tener un país más sano en lo que se refiere a la cultura de la paz.

Uno de los grandes principios de la cultura de la paz es aprender a vivir juntos, hagámoslo en beneficio de cada una de las pequeñas sociedades donde se habita y esto tendrá un impacto a nivel nacional e internacional. Promover la ética en todos los sectores de la población, dará como resultado una sociedad con respeto a la diversidad, al derecho y a la paz.

El restablecimiento de la dignidad y la autoconfianza en que le individuo puede interactuar y autodeterminar su destino en libertad, conlleva a una reconstrucción de su identidad y a un ajuste positivo en su cosmovisión.

Un ser humano que vive en dignidad goza de sus derechos humanos, civiles y políticos, promueve el bien común y se libera del devenir del caos emocional provocado por el conflicto y la falta de paz. Y como última instancia el ser humano que vive en dignidad es más propenso a la felicidad

\section{Bibliografía}

Albornoz, O. (1984). La Familia y la Educación del Venezolano. Ediciones Biblioteca Caracas. Págs. 49, 89 
Beck, A. (2003) Prisioneros del odio. Las bases de la ira, honestidad y violencia. Barcelona: Paidós

Cabello Tijerina, P., Vázquez Gutiérrez, R. (2018) Cultura y educación para la paz. Una perspectiva transversal. México Tirant lo Blanch. Pág. 45

Cabello Tijerina, P.A., Carmona Valdés, S.E., Gorjón Gómez, F.J., Iglesias Ortuño, Emilia., Sáenz López, K.A.C., Vázquez Gutiérrez; R.L. (2017) Cultura de paz. México. Editorial Patria

Cabello Tijerina, Paris, Alejandro. (2012). La mediación como política social aplicada al fortalecimiento de la cultura de paz en México y España. Tesis doctoral en intervención social y mediación. Universidad de Murcia.

Campanario, Juan Miguel y Otero, José C. (2000). Más allá de las ideas previas como dificultades de aprendizaje: las pautas de pensamiento, las concepciones epistemológicas y las estrategias metacognitivas de los alumnos de ciencias. Grupo de Investigación en Aprendizaje de las Ciencias. Departamento de Física. Universidad de Alcalá de Henares. Alcalá de Henares. Madrid.

Colom, A.J. Y Melich, J.C. (1997). Después de la modernidad. Nuevas filosofías de la educación._Barcelona: Paidós

Galtung, Johan. (1998). Cultura de paz y gestión de conflictos, Primera edición, Paris 1998, 403 páginas.

García Longoria Serrano, M. (2009). Educación Familiar. El aprendizaje de la resolución de conflictos. En J. Paradas Navas, \& J. González Ortiz, La familia como espacio educativo (págs. 161-173) Murcia: Espigas

${ }^{1}$ González Ballesteros, L., Sainz Martin. A., Hens Muñoz, M., \& Sánchez Corral. (1993) Signos y cultura de violencia. Una investigación en el aula. Córdoba: Servicios de publicaciones de la Universidad de Córdoba

Kant, I. (1993). La metafísica de las costumbres._Barcelona: Altaya.

Ledrach, J.P. (2000) El abecé de la Paz y los Conflictos. Educación para la paz. Madrid Catarata

López Gómez, Óscar «Pas e Sosyego»Un argumento de acción política en la castilla bajomedieval. Medievalismo: Boletín de la Sociedad Española de Estudios Medievales, ISSN 1131-8155, N. ${ }^{\circ} 16,2006$, págs. 41-72

Mockus, Antanas. (1994). -La educación para aprender a vivir juntos, convivencia como armonización de ley, moral y cultura-. Perspectivas, vol. XXXII, N. ${ }^{\circ}$ 1, marzo 2002. P. Ver: 
Boulding Kenneth. Paz estable entre las naciones: un proceso de aprendizaje. Revista Cuadernos de Ciencias Económicas y Empresariales, N. 26, 199

Muldoon, Brian. (1998) El corazón del conflicto. Ed. Paidós, España. pág. 63.

Munduate Jaca, L., \& Medina Díaz, F (2006) Gestión del conflicto negociación y mediación. Psicología, Pirámide

Muñoz Muñoz, F., López Martínez, M. (2004) Historia de la paz, en B Molina Rueda, \& F. Muñoz, Manual de Paz y Conflictos (págs. 42-65) Granada: Eirene

Perkins, D. (2001). La escuela inteligente. Del adiestramiento de la memoria a la educación de la mente. España: Gedisa. Pág. 18

Ramos, M. (2000). Programa Para Educar en Valores. La educación que transformará al País. Editorial Paulinas. pág. 55

Regué Barrera, M. (2015) La química de la empatía. Revista Moleqla No. 12 ISSN 21730903 Editado el 21 de marzo de 2015.Universidad Pablo de Olavide, Sevilla, España, p.62.

SODePaz (27de marzo de 2019). Cultura de paz. Obtenido de Cultura de Paz: http:/www.culturadepaz.info/home.php

Sotelo, Elena; Carretero Morales, Emiliano y Ruiz López, Cristina. Mediación y resolución de conflictos: técnicas y ámbitos. Ed. Tecnos. Madrid. Pág. 250

Toh Hin, Swee. (2002). -La educación para aprender a vivir juntos. Construcción de la paz y educación para la paz: Experiencias locales, reflexiones globales-. Perspectivas, vol. XXXII, N. ${ }^{\circ} 1$, marzo 2002.

Vázquez Gutiérrez; R.L. (2013). La mediación escolar y las habilidades sociales en los estudiantes de educación secundaria. Un estudio en institutos de la región de Murcia. Comunitania International Journal of Work and Social Sciences, 113.136.

Velasco Suárez, M., Ordoñez Acuña, B., Dávila, M.H., Roteer Hernández. L. (2012) Una educación para la paz. Programa para niños preescolares. México Trillas.

Submetido em: 22/03/2019

Aceito em: 28/04/2019 